\title{
Simulation Tools for Atom Probe Tomography : A Path For Diagnosis and Treatment of Image Degradation
}

\section{Vurpillot, Francois}

2018-12

Vurpillot , F , Parviainen , S , Djurabekova , F , Zanuttini , D \& Gervais , B 2018 , ' Simulation Tools for Atom Probe Tomography : A Path For Diagnosis and Treatment of Image Degradation ' , Materials Characterization , vol. 146 , pp. 336-346 . https://doi.org/10.1016/j.matchar.2018.04.024

http://hdl.handle.net/10138/314176

https://doi.org/10.1016/j.matchar.2018.04.024

cc_by_nc_nd

acceptedVersion

Downloaded from Helda, University of Helsinki institutional repository.

This is an electronic reprint of the original article.

This reprint may differ from the original in pagination and typographic detail.

Please cite the original version. 


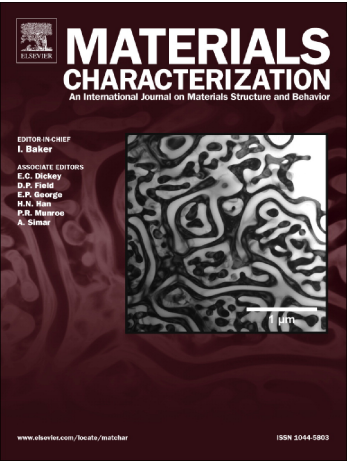

Francois Vurpillot, Stefan Parviainen, Fluyra Djurabekova, David Zanuttini, Benoit Gervais

PII: S1044-5803(18)30367-X

DOI: doi:10.1016/j.matchar.2018.04.024

Reference: MTL 9164

To appear in: Materials Characterization

Received date: 5 February 2018

Revised date: 13 April 2018

Accepted date: 16 April 2018

Please cite this article as: Francois Vurpillot, Stefan Parviainen, Fluyra Djurabekova, David Zanuttini, Benoit Gervais , Simulation tools for atom probe tomography: A path for diagnosis and treatment of image degradation. The address for the corresponding author was captured as affiliation for all authors. Please check if appropriate. Mtl(2017), doi:10.1016/j.matchar.2018.04.024

This is a PDF file of an unedited manuscript that has been accepted for publication. As a service to our customers we are providing this early version of the manuscript. The manuscript will undergo copyediting, typesetting, and review of the resulting proof before it is published in its final form. Please note that during the production process errors may be discovered which could affect the content, and all legal disclaimers that apply to the journal pertain. 


\title{
Simulation Tools for Atom Probe Tomography: A Path For Diagnosis and Treatment of Image Degradation
}

\author{
Francois Vurpillot, ${ }^{1}$ Stefan Parviainen ${ }^{1,2}$, Fluyra Djurabekova ${ }^{2}$, David Zanuttini ${ }^{3}$, \\ Benoit Gervais ${ }^{3}$ \\ ${ }^{I}$ Normandie Univ, UNIROUEN, INSA Rouen, CNRS, GPM, 76000 Rouen, France \\ ${ }^{2}$ Department of Physics and Helsinki Institute of Physics, University of Helsinki, Finland \\ ${ }^{3}$ Normandie Univ, ENSICAEN, UNICAEN, CEA, CNRS, CIMAP, 14000 Caen, France
}

The ideal picture of a near-perfect 3D microscope often presented regarding Atom Probe Tomography faces several issues. These issues degrade the metrological performance of the instrument and find their roots in the phenomena acting at the atomic to the mesoscopic level in the vicinity of the surface of a field emitter. From the field evaporation process at the atomic scale, to the macroscopic scale of the instrument, the path to model the imaging process and to develop more accurate and reliable reconstruction algorithms is not a single lane road. This paper focused on the numerical methods used to understand, treat, and potentially heal imaging issues commonly affecting the data in atom probe experiments. A lot of room for improvement exists in solving accuracy problems observed in complex materials by means of purely electrostatic models describing the image formation in a classical approach. Looking at the sample at the atomic scale, the phenomena perturbing the imaging process are subtle. An examination of atomic scale modifications of the sample surface in the presence of a high surface electric field is therefore mandatory. Atomic scale molecular dynamic models integrating the influence of the high surface electric are being developed with this aim. It is also demonstrated that the complex behavior of atoms and molecules in high fields, and consequences on collected data, can be understood through the use of accurate ab-initio models modified to include the impact of the extreme surface electric field.

Keywords : Atom Probe Tomography, Modeling approaches, Molecular Dynamic, Ab-Initio, nanometrology 


\section{Introduction}

The capability to correlate the physical properties of materials to the local three dimensional distribution of atoms is nowadays crucial in designing new and improved materials and devices. When designing nanostructured materials, the dream is to be able to catch with the highest metrological performance a snapshot of the region of interest. Being able to identify structural and elemental information with 3D details on the single-atom level could transform the understanding of the relationship between structure (e.g. composition, defects, crystallography) and properties (e.g. magnetic, electric, photonic, or mechanical properties for instance) at the most fundamental level. This approach is explored using the atom probe tomography instrument (APT). This instrument is indeed unique in the world of nano-analysis tools, for its ability to provide, after analysis of a volume of interest in the material, a 3D map of atom positions, with atoms labelled by their elemental identities [1, 2, 3, 4].

By wandering between the static positions of atoms present in an APT dataset, the instrument's user often feels that the three dimensional image is a snapshot of the specimen that was left unharmed by the analysis process. This feeling is obviously an illusion. At the APT specimen level, i.e. a sharply pointed needle (called the tip), the imaging process utilizes extremely intense physical mechanisms on the surface and sub-surface of the materials. A strong electric voltage generates locally a huge surface electric field (several tens of $\mathrm{V} / \mathrm{nm}$ ). This electric field gives rise to a Maxwell stress on the extreme surface, pulling atoms outwards, up to the bonds breaking. Atoms are eroded one by one through this mechanism. A delicate control of the erosion rate is mandatory to keep intact the sample during the whole analysis process (at least during the analysis of the region of interest). Indeed the mechanical stress on the surface approaches the yield strength of the material of interest. The basic erosion mechanism is to induce sufficient atomic motion so that the surface atoms escape the polarized sample. The motion of the atom is accompanied by severe charge rearrangement by which it can lose one or more electron (this process referred to field ionization takes place in the first nanometer of the trajectory). The full mechanism (field- 
induced bond breaking of surface atoms, and field ionization) is called field evaporation. Ions are instantaneously accelerated by the voltage gradient existing in the vicinity of the needle surface and are rapidly expelled into the vacuum. These ions follow a trajectory from the initial atomic positions to a macroscopic detector.

The position-sensitive time-resolved detector records ion hit positions (corresponding to the $\mathrm{X}$ and $\mathrm{Y}$ axis) and also the time-of-flights (corresponding to the mass-to-charge ratio, i.e. ion identity) of ions. With this information, the analyzed volume can be reconstructed and represented using a 3D atom map. The reconstruction method uses the simple back-projection principle to deduce the ion trajectories. This apparent simplicity in APT is based on the deterministic and classical nature of the trajectories from initial positions of frozen atoms to the impact of the projected ions. These trajectories are only influenced by the diverging electric field distribution in the vacuum between the sample and the detector (more details about the basics physical principles can be found in [1-4])

It is important to characterize the metrological performance from the reconstructed images, to compare this particular instrument to other instruments used in materials science such as transmission electron microscopy or scanning electron microscopy. We will focus on spatial parameters and composition measurement parameters i.e. "resolution", "precision" and "accuracy". Spatial resolution is "the minimum feature that can be observed". In classical microscopes the spatial resolution depends on the size of the pixels in the camera. In APT, atom positions are represented by 3 floating values $(\mathrm{x}, \mathrm{y}, \mathrm{z})$ for each atom and there is no pixel size. Each position is the result of a single experiment. As a result, the use of spatial resolution for the set of points is awkward; we will refer to spatial precision instead. Note that spatial resolution can, however, be used in composition maps or density maps. Spatial precision is the random error in the measurement of a position in a single experiment. The spatial precision can be estimated by 
repeating a large number of experiments. The dispersion of the values around the average value gives an estimate of the precision. Spatial accuracy corresponds to a systematic distortion of the position measurement. Spatial accuracy may affect strongly the precise absolute measurement of large-scale distances.

When producing an image composed of a set of points, the optimization of the reconstruction is evaluated using accuracy and precision. An accuracy degradation produces image distortion. Optimizing accuracy consists in knowing precisely the transfer function between the actual position and the detected position. This can be done only if the transfer function between the measured position and the real position remains bijective. Several solutions of reconstructed positions are therefore possible and only maximum likelihood methods can help to correct the final image.

Spatial precision degradation induces image blurring. Note that image restoration methods can be used to improve the image when sufficient information exists in the image. Thus, spatial precision in the positioning of single atoms in the reconstructed image may vary from a fraction of an angstrom to several nanometers. Spatial accuracy can be as good as a few angstroms over several hundreds of nanometers (better than $0.1 \%$ relative accuracy) to several tens of nanometer (10\% relative accuracy). This variation in metrological performance is in most cases not due to the user, or to the physical parameters of the instrument, but linked to the mechanisms of gradual erosion of the sample during the analysis process. A diagnostic of the image is necessary to evaluate the confidence in the results obtained for the instrument. A correct diagnostic can sometimes only be achieved by fine scale inspection of the mechanisms at work during the analysis. Ad-hoc simulation tools have been developed for years to first understand the analyzing process, and to find usable solutions to improve metrological parameters. Different simulation tools were built up for the purpose of understanding different issues and shortcomings $[5,6,7,8,9,10$, $11,12,13,14,15,16]$. We will focus this paper on three different kinds of modelling approaches used to 
diagnose different kinds of sicknesses in APT. Spatial precision and accuracy problems in metals and alloys can be understood by looking at fine scale effects during the field evaporation process. Some of these problems are due to the dynamic evolution of the surface morphology of the sample induced by field evaporation. In this case, simple models based on a succession of static descriptions of the tip are sufficient [5-16]. More refined models are now developed to understand how the tip evolves between successive evaporation using molecular dynamic approaches $[17,18,19]$. Some examples will be presented below. Finally, some issues can only be addressed by considering the quantum nature of atoms and ions, which determine the potential energy of interaction between the atoms and the surface, which in turns governs the field evaporation process. Some new approaches are presented to interpret common composition and spatial precision biases observed experimentally in the analysis of oxides and nitrides [20,21].

\section{Methods}

\section{Which model for which symptom?}

Reconstructed atom maps are deduced from the ordered list of impacts coordinates of ions as a result of the field evaporation of the sample. In APT, the ion projection is directly related to the geometry of the specimen. This is not the norm for most microscopy techniques, and as the specimen is progressively analyzed, the surface may evolve in a complex manner. The consequence of this evolution can be global. Beyond a distance corresponding to a few radii of curvature of the specimen, the ion trajectories are influenced by the meso- and micro-scale parameters, such as the average radius curvature of the specimen, its shank angle, and the overall electrostatic environment of the specimen (i.e. presence of a substrate, distance to an extraction electrode, etc.) [1,5]. Deviations of ion trajectories may also arise from atomicscale variations of the specimen surface that may result in distortions in the reconstructed image. Understanding of how the image is reconstructed in 3D requires understanding how the sample shape 
evolves due to erosion process. Modeling the imaging process in APT is linked to our ability to model how the sample is eroded, atom by atom [8].

Nevertheless, a universal tool that brings understanding and solutions to the reconstruction artefacts in APT does not exist yet. The complexity of the problem comes mainly from the large range of spatial scales that must be handled by the model. A full-scale simulation of the analysis of an APT sample means understanding the trajectories of billions of atoms from the atomic scale to the macroscopic scale. We deal also with several timescale ranges, from the first steps of erosion that require femtosecond resolution, to the microsecond time scale of a single ion flight, and the hour/day time scale of the full analysis process. To deal with these constraints, a number of simplifications have been applied to focus the simulation tools on a specific kind of expected artefact, a material, or a specimen geometry. We may also keep in mind that the algorithm used to reconstruct APT datasets is different when calculating the lateral positions (induced by ion projection) and the depth positions (assuming conservation of volume from collected atoms). Let us recall that the lateral position of an atom is obtained from the impact position $(\mathrm{X}, \mathrm{Y})$ on the detector $[1-4,22]$. A very first approach is simply to consider a constant magnification $\mathrm{M}$ (M 1 million), so that $(\mathrm{x}, \mathrm{y})$ position on the tip is found as $\mathrm{x} \sim \mathrm{X} / \mathrm{M}$, and $\mathrm{y} \sim \mathrm{Y} / \mathrm{M}$. It is worth noting that with a good calibrated local value of $\mathrm{M}$, lateral precision performances are insensitive to this over-simplification. The depth position (z position) is obtained from the volume conservation aspect between two successive atoms. The depth increment is given by $\Delta \mathrm{z}_{1}=\mathrm{V}_{\mathrm{at}} /(\mathrm{QSa})$, with $\mathrm{Q}$ the detection efficiency, and Sa the surface of the probed zone of the sample. This increment is less than a picometer in most cases, and is our intrinsic instrumental depth precision.

\section{Depth precision}

In figure 1a, the ideal order of field evaporation as it may be expected in APT image reconstruction is demonstrated. In reality, there will be processes that confuse this order. For instance, two mechanisms may be expected to be responsible for the degradation of depth precision in APT reconstructions, shown 
in figures $1 \mathrm{~b}$ and $1 \mathrm{c}$. First the order of evaporation can be perturbed during the erosion process (figure $1 \mathrm{~b}$ ). Models must describe how the atomic surface evolves under the presence of a high electric field, which depends on the local roughness at the atomic scale, between the successive evaporation of two atoms. We may note that assuming atoms remain at rigid positions on the tip surface is sufficient only if spontaneous field evaporation of atoms is involved. Nevertheless, the impact of the local arrangement of atoms, underpinning the local electric field, and the influence of the stochastic nature of the field evaporation process are important ingredients of recent models developed to mimic the erosion of alloys. Understanding depth precision degradation in metallic alloys under high temperatures requires more elaborate models describing the interaction of mobile atoms (figure 1c). Only a molecular dynamic approach may be sufficient in this case $[18,19,23]$. In non-metallic alloys, the problem can be even more complex, since electric charges redistribute in a complex way depending on the chemical nature of the atoms. The surface may rearrange itself to minimize the local energy. In this case, only a quantum model of the surface, that includes the presence of the local high electric charge, could give some valuable information [23]. 
(a) Depth precision :

Ideal sequence of evaporation
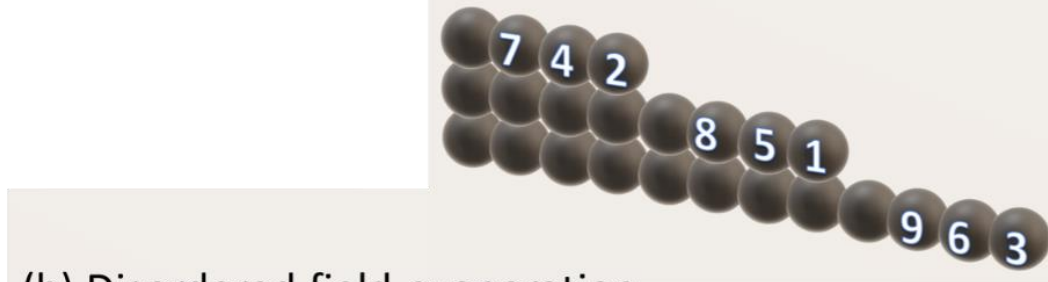

(b) Disordered field-evaporation

(c) Diffusion prior to field-evaporation
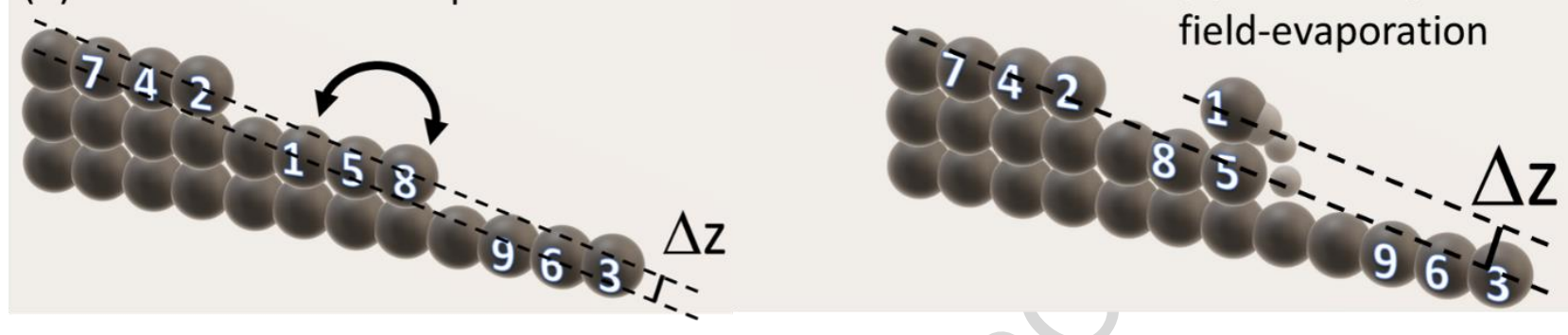

Figure 1: Possible physical mechanisms responsible for precision degradation of the reconstructed depth position; (a) ideal case. The order of evaporation is indicated by the sequence of numbers. (b)Degradation induced by a change in the order of evaporation. A change in evaporation order results in a degradation $\Delta z$ of the depth precision (c) Degradation induced by atomic movement of atoms prior to the evaporation process. Pre-evaporation displacements perturb significantly the evaporation order with direct consequences on the depth precision.

\section{Lateral precision}

Lateral precision degradation is much more complex since it involves the trajectory of atoms from its initial position on the surface to the final impact on the detector. Most of the trajectory is the result of the acceleration in the smoothly varying electric field in the space between the tip and the detector and is, therefore, completely deterministic. Nevertheless, the trajectory is very sensitive to the velocity (intensity and direction) during the initial stage of the flight. This was experimentally demonstrated in field ion microscopy for instance [24]. The diverging distribution of the electric field acts as an amplifier and must be integrated in the modelling process. 
(a) Ideal ion trajectory
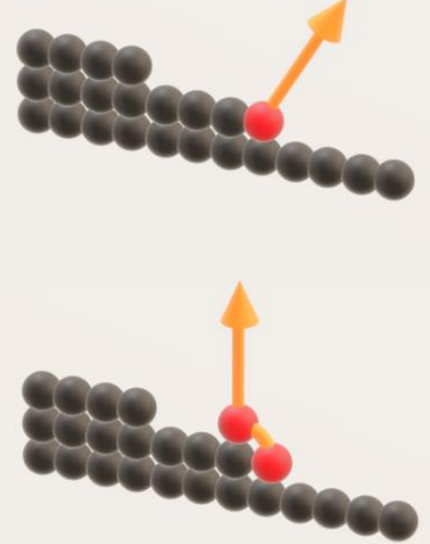

(d) Rolling-up motion (b) atomic diffusion degradation
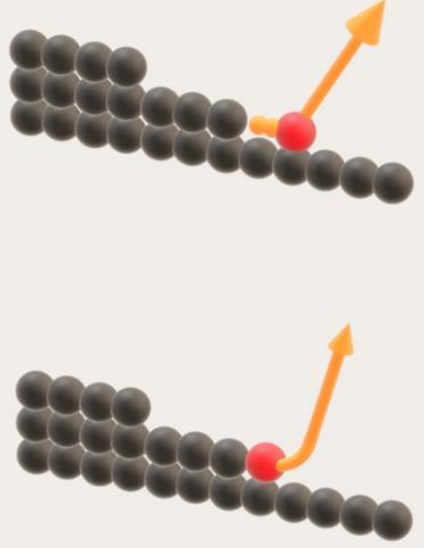

(e) Trajectory aberrations (c) Initial velocity of ions
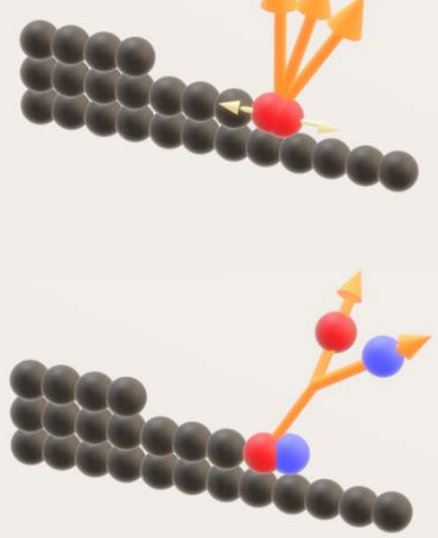

(f) In flight dissociation

Figure 2 :Possible physical mechanisms responsible for degradation of the lateral position in reconstructed dataset;(a) ideal trajectory (b) lateral position is perturbed by atomic hopping at the surface induced by surface diffusion prior to field evaporation (c) influence of the initial lateral kinetic energy $(d)$ reconstruction process at the moment of the evaporation (rolling-up). Atoms moving to a metastable position in the very first step of field evaporation (e) trajectory deviations induced by variation of the electrostatic environment, named trajectory aberrations $(f)$ in flight dissociation of molecules, liberating kinetic energy release.

The best lateral precision achieved experimentally approaches $0.2 \mathrm{~nm}$, a value close to the ultimate interatomic distance [25]. However, this is not the general case. Since precipitates as small as 1-2 nm are commonly observed experimentally, we generally assume that the lateral precision is below or close to 1 $\mathrm{nm}$. Considering the reconstruction process, several physical mechanisms have to be considered in the interpretation of the degradation of the resolution.

These mechanisms are represented in figure 2 and are listed below. For comparison, the ideal case of atom detachment due to field evaporation is shown in figure 2a. 
- Surface diffusion prior to field evaporation (figure $2 b$ )

Even if the temperature of a sample tip is low, a probability that an atom at the tip surface will perform a diffusion jump still exists [26]. This effect depends on the energy barrier for surface diffusion compared to the barrier for field evaporation and the residence time of a surface atom prior to field evaporation. The value of the surface surface diffusion barrier $\mathrm{Q}_{j u m p}$ is usually between 0.15 and 1.1 $\mathrm{eV}$ for most materials. According to these values, a simple calculation of the diffusion probability shows that the residence time of an atom before evaporation is much shorter than the residence time on its atomic site before diffusion. Surface diffusion is relevant only in particular cases, when very small diffusion barrier exists at the tip surface, or if the analysis is performed at a high temperature [27]. Such a high temperature rise is induced in APT experiments working in a laser mode. However, this increase is only transient and lasts only during a very short pulse [45]. Due to short pulse durations and rather low laser energies used, the temperature rise is not significant and only very high laser energies can generally induce significant surface diffusion. We also note here that the diffusion process can be different if the strong gradients of high surface electric fields are present on the surface. Modelling of this process requires integration of the impact of the electric field by classical Kinetic Monte Carlo (KMC) models, or Molecular dynamics (MD) models which are less practical for this purpose as they limit simulations to a nanosecond timescale only

- Initial transverse velocity of evaporated atoms (figure 2c):

The early work of Chen and Seidman proposed this mechanism as one of the sources of blurring in Field Ion Microscopy [24]. In the initial state of the flight, an evaporating atom has an intrinsic (vibrational) velocity that is derived from the thermal energy. This velocity is small, but nonzero and gives rise to a lateral blurring of the impact position onto the detector. Considering the sample temperature, this thermal velocity is predicted to cause a lateral dispersion less than $0.1 \mathrm{~nm}$ which is rather small and can be neglected. 
- Rolling-up motion (figure 2d):

The classical field evaporation theory assumes that a field-evaporated atom follows a trajectory normal to the surface (figure 2a). Distortions in desorption maps of pure metals led some authors to assume that the paths of evaporated atoms may be significantly altered by the interaction with neighbour atoms in the initial stage of the flight. Analysing the experimental dependence of evaporation field on temperature for (110) surfaces of a number of metals (including Al), Wada [28] concluded that direct field evaporation of atoms (through the image-hump, or the charge-exchange model) are unable to produce satisfactory agreement. Wada suggested an alternative route for surface atoms to evaporate: instead of escaping directly from their original position, the atom undertakes a short trip (similar to diffusion process) to locate itself where evaporation is easier. In particular, a jump of a kink atom atop a step edge was considered in [28]. The above mechanism implies only a short-range diffusion such as a jump to a meta-stable site (a surface atom diffuses for example, to the edge of a tip plane). The field there might be sufficient for the atom to leave the tip straight away. Only molecular dynamics simulations can be used to model these mechanisms. This mechanism was named rolling-up motion by Waugh because rather than leaving directly the surface, the atom experiences a path by rolling around one of its neighbour atoms to reach a position where it can be lifted out from the surface [29]. Sánchez and Lozovoi compared theoretically the energy barriers for an atom on a (111) Al surface under very high electric fields to be field evaporated or to diffuse from one hollow site to another via a bridge site or atop of the neighbour atom [30]. The authors concluded that the barrier for a jump over a neighbouring atom remains rather high, even if high electric fields are applied, while the barrier for a jump via the bridge site almost disappear at very high fields. Even at smaller fields, the diffusion barrier is considerably smaller than the one for the field evaporation event. Hence, the mechanism, which combines diffusion and evaporation events, can indeed compete with the direct field evaporation mechanism. In dilute alloys, directional surface diffusion can be observed even at low 
temperature. Species like phosphorous, nitrogen, or carbon, are observed to move preferentially toward specific crystallographic locations. This behaviour is not general and is not well understood at this moment. This kind of movement induces artificial chemical gradients in a 3D volume [31].

- Trajectory aberrations in the first steps of flight (figure 2e)

The strong magnification in APT is achieved through the high curvature of the tip apex, where the electric field is strongly enhanced. Due to the radial distribution of the field around the tip apex, the ions (evaporated atoms) fly radially from the surface of the sample to the detector. The distance between the ions, which may have been very closely positioned on the surface, increases in a way that depends on the distance from the sample to the detector. However, during the initial stage of flight, the ions are accelerated in the local electric field, which is non-uniformly distributed not only around the apex of the sample tip as a whole, but also around the local neighbourhood of the atoms before they leave the surface. Any deviation in trajectories very close to the surface will be strongly magnified by the same mechanism. If we consider an atom in a given lattice position on the surface of the tip, the trajectory of this atom is deterministic and follows a path induced by the distribution of surrounding atoms that builds the local field distribution. Models must integrate the distribution of the electric field in the first steps of flight as well to reduce the aberrations $[6,19,32,33,34,35]$. By assuming atoms are fixed on a lattice, the impact of the temperature or the presence of species with different evaporation fields strengths were evaluated by field evaporation models. Note that local variations of ion trajectories induced by changes in evaporation order were experimentally observed in field ion microscopy [32]. Thus, the correct input of the field distribution after each evaporated atom should improve APT reconstructions.

- In flight dissociation of molecules (figure 2e)

In oxides or nitrides, the ions are often emitted from the surface as molecules, rather than single ions. Some molecules are not stable in strong fields, which are further enhanced near the tip surface, and 
may dissociate into separated single ions. During this process, some energy is released, which is transferred to the ions as kinetic energy with a randomly directed velocity. This process will induce significant spread in the lateral positions of the registered ions. A correct interpretation of the lateral position degradation induced by this effect requires accurate modelling of the dissociation process $[20,21]$.

\section{Modeling dynamics of field evaporation using electrostatic models}

\section{Basic principles of the field evaporation models}

Most of the artefacts observed experimentally in the reconstruction of APT datasets are reproduced using models that assume the electric field distribution with electrostatic approximations on a field emitter [5, 8]. The compromise of these models is to consider the sample being un-deformed, and non-perturbed between two successive removals of surface atoms under the effect of the static electric field. This hypothesis is supported by experimental conditions during an atom probe experiment. The sample is held at a cryogenic temperature in the range $20-80 \mathrm{~K}$. From a classical point of view, atoms are frozen, and atomic movements are limited. The recent application of the Atom Probe to non- metallic materials have further increased the complexity of mechanisms that must be taken into account to understand the image formation in APT [13, 23].

To understand how the electric field may affect the surface atoms, and the way the tip is eroded during atom probe analysis, it is important to determine how the electric field is generated locally. Therefore, the exact relationship between the curvature at the atomic scale and this field (which results simply from the distribution of surface charges) must be evaluated. This distribution is a key parameter to understand the regularity of the surface erosion by field evaporation of atoms. Most advanced treatments use density 
functional theory (DFT) to calculate at the atomic scale how electron density redistributes when a sheet of materials is biased to a constant voltage. These models, which reproduce the atomistic surface of the material, ought to give the most reliable results. However, DFT models are limited to a few hundred atoms at maximum and are unable to give a complete picture of a biased tip at the quantum level $[23,30,36,37$, 38]. To simplify the problem, two alternative ways of computation have been pursued.

A quasi-conductive material may be considered as a continuous medium down to the atomic scale (figure 3). Charge is found to concentrate on surface atoms. Inside the volume, quantum calculations predict that the electric field rapidly diminishes within a distance smaller than the size of an atom, and only residual oscillatory contributions arise in a few subsequent layers. DFT calculations predicts that the most protruding atoms concentrate the strongest surface charge, and, are therefore the locations of the highest external field (figure 3a) [36-39]. This result justifies the use of more rough calculations or modelling approaches that approximate the atoms of the tip surface by a subatomic scale polyhedral representation (figure 3b), as surface charge distributed in polyhedron produces a field that is strongly dependent on the local surface curvature. Each atom produces its own protrusion at the tip surface, and so the local field differs strongly from the average field above the surface. Surface atoms may be considered as small geometrical volumes which can be as simple as cubes, with charges present on the external facets of these volumes. The field distribution in the free space may be calculated assuming the external morphology of the assembly of atoms, and solving the Laplace equation assuming a constant voltage $\mathrm{V}$ on the sample, and a lower voltage on the electrode above [8]. A 3D mesh of voltage nodes, fined-grained close to the tip surface, and coarse-grained at larger distances can be used to estimate with good precision the entire electric field distribution. This mesh is used to compute individual ion trajectories travelling from the initial tip surface to the detector. Different mesh symmetries, mesh geometries, boundary approximations, and computational optimizations were developed by different authors for this purpose, and were used to 
understand the image formation in APT. We refer the reader to [8] for a complete overview of the evolution of modeling approaches.

An alternative approach for simulating the field evaporation process in Atom Probe Tomography uses the electrostatic Robin's equation to directly calculate the charge distribution over the conducting surface of the tip, requiring no knowledge of the surrounding electric field (figure 3c)[7]. The partial ionization state of the surface atoms is at the core of the method. The surface charge is assumed here to be discrete and spread on the positions of every surface atoms. The field distribution is retrieved using Coulomb's law, based on the distribution of point charges, without the need for a supporting mesh. The only requirement is a fine scale definition of surface atom positions, and the normal direction to the surface on these points (n). The computational efficiency is ensured by an adapted version of the Barnes-Hut N-body problem algorithm. More technical details about the method could be found in [7]. 


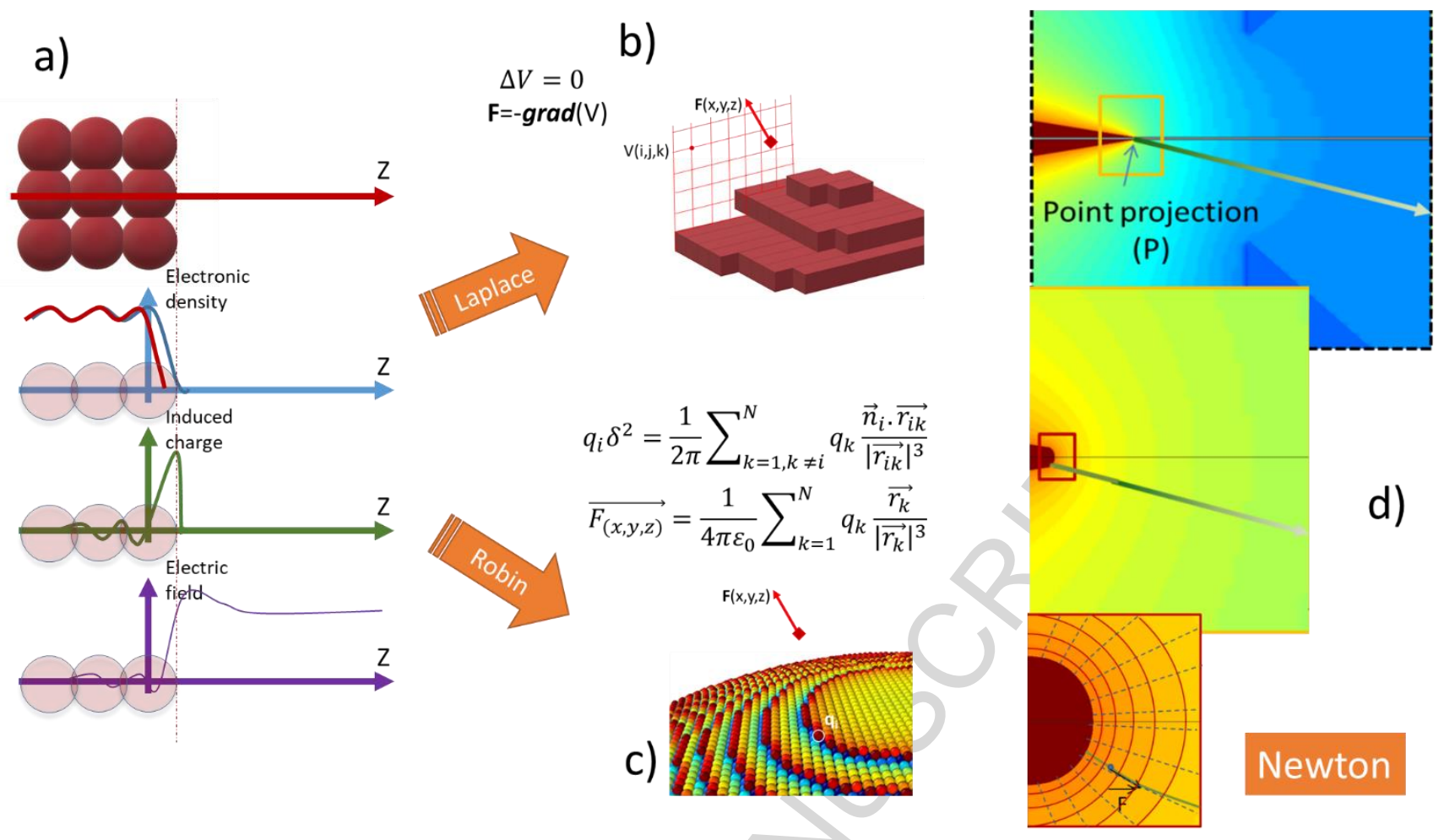

Figure 3: (a) Field evaporation assuming a quantum model of atoms, the application of a voltage on the sample changes the electronic density (from blue curve with no voltage to in red curve with an applied voltage). Electronic density is slightly shifted inside the materials which gives rise to a positive effective charge localized on the surface atom (in green) and the local electric field $\boldsymbol{F}$ (in purple). This mechanism is modelled through simple approximations of the (b) Laplace equation (c) Robin equation, which enables to determine ion trajectories (d) from the resolution of the Newton equations.

Once the potential, or the distribution of surface charges for the initial input structure has been obtained, the dynamic desorption of atoms is simulated by repetitive execution of three basic steps: First, the algorithm looks for the surface cell which is exposed to the maximum field strength and removes it, i.e. the particular bulk cell becomes a "vacuum" cell. Different field evaporation thresholds for desorption are taken into account by appropriate scaling of the surface field. The sequence of evaporation is then deduced by assuming some simple physical law describing the order of evaporation. The simplest one assumes that 
the sequence is fully deterministic, the atom submitted to the highest field being the first to be removed from the tip surface. Second, the potential is recalculated for the new configuration. And third, the iontrajectory starting from the origin of the removed atom is computed by integration of Newton's equation of motion.

\section{Role of the evaporation process in the spatial precision}

\section{degradation}

As described previously, computation of ion trajectories is completely deterministic, as soon as initial velocities of evaporated atoms are null, which is valid considering a cryogenic sample temperature. Nevertheless, it was found that the process of field evaporation might induce slight degradation of the spatial precision. Indeed, without a field atoms are confined in a potential well, with an adhesion energy proportional to the sublimation energy of the material. In the presence of a surface electric field, this potential well is deformed, so that an energy barrier $\mathrm{QEV}_{\mathrm{EV}}(\mathrm{F})$ smaller than the adhesion energy exists and may be overcame with sufficient activation energy. If so, the process can be considered as thermally activated [1-4]. In other words, the probability of an atom to be field evaporated can be considered based on Boltzmann statistics. Within this approach, an Arrhenius equation can be written to describe the evaporation rate $\mathrm{k}_{\mathrm{EV}}(\mathrm{F}, \mathrm{T})$, as a function of the specimen temperature $\mathrm{T}$ and the electric field $\mathrm{F}$. This equation reads as $\mathrm{k}_{\mathrm{EV}}(F, T)=A e^{\frac{-Q_{E V}(F)}{k_{B} T}}$, with $\mathrm{k}_{\mathrm{B}}$ the Boltzmann constant and $\mathrm{A}$ the rate constant prefactor or surface-atom vibration frequency. $Q_{E V}(F)$ decreases monotonically with $F$, and vanishes for a threshold electric field named $\mathrm{F}_{\mathrm{EV}}$, the evaporation field of the atom. This threshold field depends on the local atomic environment [40], but is often taken as a material-dependent constant for simplicity. When crossing the barrier, the atom is rapidly ionized. Close to the evaporation field $\mathrm{F}_{\mathrm{EV}}$, the barrier height (a small fraction of an $\mathrm{eV}$ in the pre-evaporation stage) decreases continuously with $\mathrm{F}$. The evolution of $\mathrm{QEV}_{\mathrm{EV}}$

with $\mathrm{F}$ is often simplified to the linear relationship $Q_{E V}(F)=C\left(1-\frac{F}{F_{E V}}\right)$, where $\mathrm{C}$ is an energetic 
coefficient [3]. Though very dependent on the surface field (a 1\% field variation changes the evaporation rate by a factor of 10), the stochastic nature of field evaporation process may change the order of evaporation events, with direct consequence on the depth precision, and indirect consequence on the lateral precision (through variations of the local atomic environment of the evaporating atom).

These degradations were investigated by implementing a kinetic Monte Carlo (KMC) approach to address the dynamic choice of the evaporation order as a function of the gradual evolution of the electric field at the tip surface $[8,9]$. In this model, the evaporation sequence is determined step by step, as explained above. The rate $k_{i}$ is considered as the transition frequency between the current state of the surface (all atoms being part of the surface) and the next state, (the surface with the selected atom field evaporated). A KMC method is used to choose the order of evaporation. For each step, $k_{i}$ values are calculated for all surface atoms and the atom to be evaporated is chosen via a random process. In this case, the probability distribution of the surface atoms depends on the temperature. Using a residence time algorithm, one atom is evaporated at each step regardless of its absolute probability per unit of time to leave the surface. The choice of the evaporated atom is obtained by calculating the relative rate of evaporation $k_{i}^{r}=\frac{k_{i}}{\sum_{\text {surface }} k_{i}}$ for each atom. A given atom is therefore defined by a part of segment of length $k_{i}{ }^{r}$; This length gives a relative probability of field evaporation of this atom. A pseudo random number $P_{r}$ comprise in the interval $[0,1]$ is generated, so that the atom $j$ with $\Sigma_{i=1}^{j-1} k_{i}^{r}<P_{r}<\Sigma_{i=1}^{j} k_{i}^{r}$ is chosen as the atom to be field evaporated. This process takes into account both the distribution of electric field at the tip surface and the stochastic nature of the field evaporation. In order to mimic realistic experimental conditions, the overall electric field can be adjusted to keep a constant evaporation flux $\Phi_{\text {fixed }}$ close to the experimental value.

This degradation is demonstrated in figure 4 . A face centred cubic sample, with the constant $\mathrm{C}$ taken to be $1 \mathrm{eV}$, is field evaporated using the $\mathrm{KMC}$ model. The temperature was varied in the range $10-500 \mathrm{~K}$. Figure 4a shows the image of impacts on the detector for three temperatures of 10,150, and $300 \mathrm{~K}$. The visual 
deterioration of the lateral position with $\mathrm{T}$ is obvious, with a blurry image generated at high $\mathrm{T}$. The stochastic nature of the field evaporation degrades gradually the regularity of images. A measurement of the depth precision was obtained by measuring the width of reconstructing (001) atomic planes in figure 4b (using Fourier method described in Ref. [25]). The atomic planes reconstruction is degraded, in reasonable agreement with depth precision measurements obtained in pure metals. The impact on the lateral position is even more pronounced, as seen in figure 4c. Nanometre lateral dispersion is induced by the disorganized morphology of the tip surface inducing random trajectory aberrations for evaporating atoms. This is possible because of the dynamic change of the surface of the field emitter. One atom can be suddenly submitted to a high field after the field evaporation of one of its neighbours, resulting in instantaneous evaporation. 

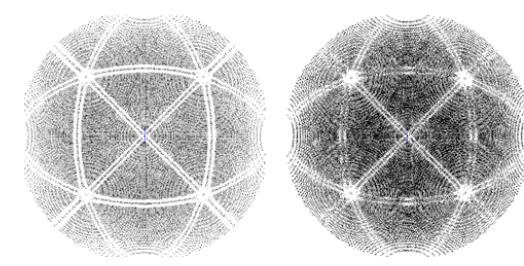

$10 K$

150K

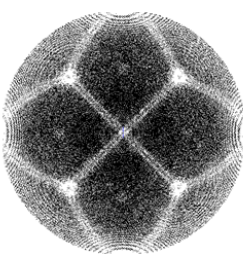

$300 \mathrm{~K}$

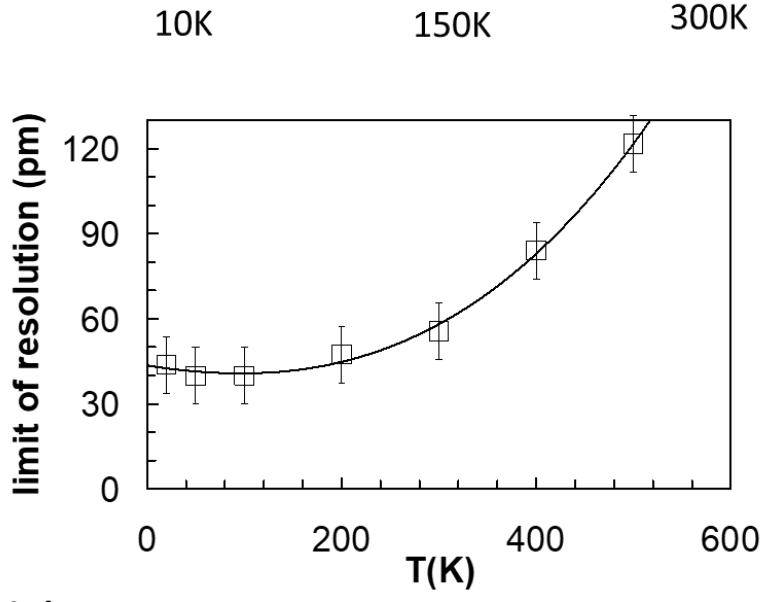

b)

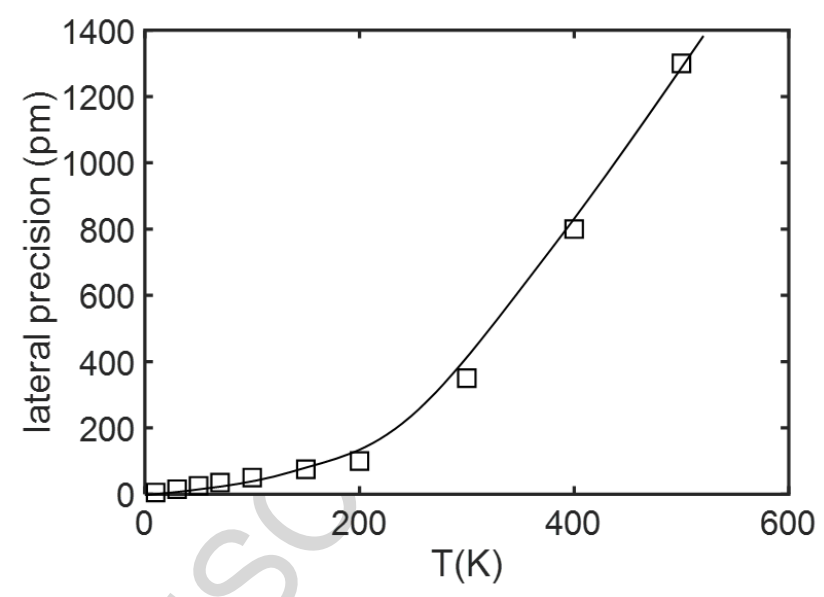

c)

Figure 4: (a) Calculated maps of ion impacts on the detector, for a simple FCC sample being field evaporated at 3 different temperatures using a KMC algorithm. (b) Calculated impact of the temperature on the depth resolution and (c) lateral precision

\section{MD - Electro Dynamic Molecular models}

The cornerstone of the dynamic field evaporation simulation approach was the introduction of an atomically defined surface of the sample. Individual deviations in trajectories and local changes in evaporation order are taken into account by these now standard methods [8]. Nevertheless, we are still far from a complete picture of the field evaporation phenomenon. It is now clear that a complete model should consider "pre-evaporation" effects such as atomic surface diffusion and global tip deformation induced by electrostatic pressure, as well as "post-evaporation" effects such as atomic relaxation. Close to the field evaporation value, the energy barrier for field evaporation of surface is extremely small, about $0.1 \mathrm{eV}$. The energy barrier for surface diffusion without an electric field is in the range $0.2-1 \mathrm{eV}$ depending on 
materials and surface structure. Increasing the temperature thus makes field evaporation much more likely than surface diffusion, andthe probability of degradation of the lateral positions by this phenomenon is increased. Under high voltage the electric field at the tip surface is highly non-homogeneous and the diffusion process is perturbed. It was observed by Tsong\&Walco $([26,41])$, that a electric field gradient may generate a directional random walk, by reducing the energy barrier for surface diffusion. Short diffusional jumps may also be influenced by local electric fields. Quantum mechanics based calculations revealed a strong modification of diffusional barriers for surface diffusion in strong external fields [29]. Hence the diffusion mechanism of evaporation can compete with the direct evaporation mechanism. Taking into account the low value of the barriers under high electric fields, these processes can be simulated by molecular dynamics methods. Although classical MD does not include electronic effects as it is done in the DFT calculations, the algorithm can be extended to include electronic effects as proposed e.g. by Djurabekova et al. [18] (and more recent developments in [41,42] appears as a promising road). In this approach, in addition to the standard interatomic interactions, the interaction between charged surface atoms and the electric field is included, as well as the Coulomb interaction between the partially charged atoms. In this approach, the charge was calculated via solution of the Laplace equation around the surface features, and utilizing Gauss' law to calculate a corresponding partial charge on each atom exposed to the electric field. Recently the Robin-Rolland model described above has also been successfully utilized [17]. This last approach is often more efficient, as only the local charge and field information is relevant when considering the morphological evolution of a structure, rather than the full electric field in the volume above the surface.

In this manner, it is possible to model efficiently and accurately the dynamic evolution of the sample specimen on the atomic level, as well as the shape of the surrounding electric field [42]. It is important to note that the shape of the sample tip, as well as the local electric field are tightly coupled together in a feedback loop[43]. A change in the sample geometry may enhance locally the electric field which, in turn, 
affects the shape of the tip via surface diffusion and tension exerted by the field at the surface. Models, such as MD, are needed to understand the morphological changes that may occur, and which affect the obtained tomographic data.

In addition to modeling dynamic processes, molecular dynamics also has another advantage in that while integrating the atomic trajectories, information about the binding energy of each atom also becomes available. This binding energy can be used to estimate the evaporation probability for individual atoms more accurately than simply looking at the local electric field strength and comparing them with pretabulated critical evaporation field values. This approach is more flexible, as it makes no assumptions about the crystal structure, number of neighboring atoms, or other factors that affect the critical evaporation field. It is also possible to estimate the critical evaporation field directly, by observing the value of the local electric field that is sufficient to break the bonds between an atom and the rest of the surface.

These simulations have been used to estimate trajectory aberrations in single and multi-phase systems [18]. In these, the atoms for evaporation were selected at random based on the value of potential energy of atoms calculated by considering the local electric field and local atomic environment, including the presence of precipitates. The MD relaxation runs between the evaporation events allow the atoms to assume energetically more favorable positions, which has a small, but visible impact on results. This might be important in some situations, especially considering that the precision of atom probes improves continuously. In particular, it was shown that when a precipitate with a larger lattice constant than the surrounding matrix is located at the surface, it may start protruding outwards due to the pressure. This protrusion, in turn, affects the local electric field direction. High-field MD has also been recently used to study the relaxation of atoms in 3D FIM, in order to explain the post-evaporation motion of atoms [17]. In this case, it was shown that MD atomic relaxation is able to take into account only a small fraction of the observed atomic motion (observed motion in FIM is tens of picometers [44], while the simulated post- 
evaporation relaxation is of the order of a few picometers). The MD model, including the dynamic distribution of surface charge, also gives access to the dynamic variation of the direction of the electric field vectors after each evaporation. It is proven that the most important factor in FIM is the change in the direction of the local electric field, due to changes in the sample geometry, affecting the image formation.

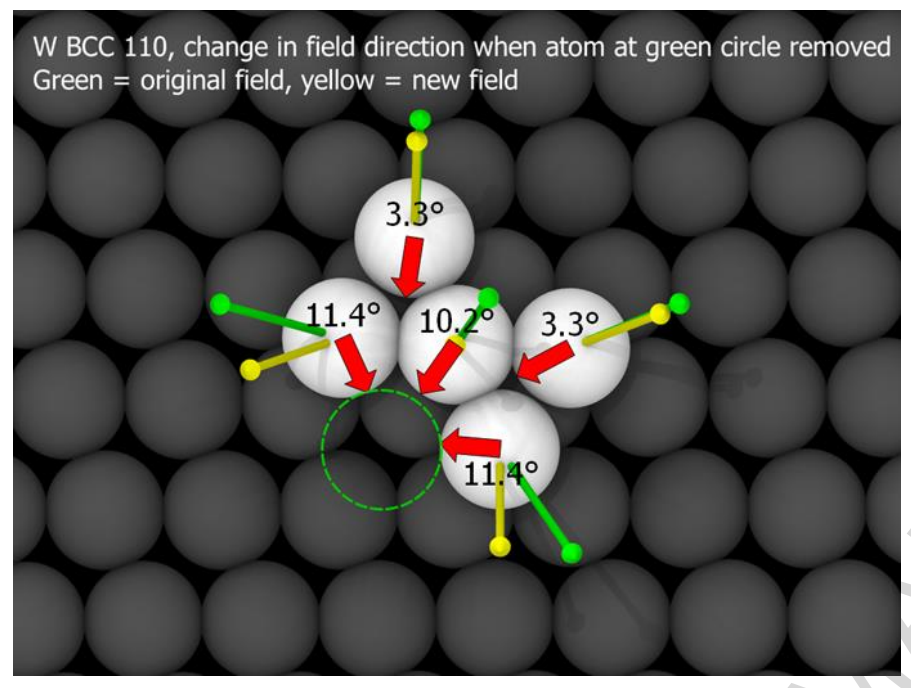

Figure 5: Change in the direction of the local electric field, following the evaporation of an atom at the green circle. Green arrows indicate the direction of the field pre-evaporation and yellow arrows postevaporation. The angles shows are the changes in the direction of the electric field and the red arrows show the direction of the change in angle.

As stated above, the migration barrier for surface ad-atom migration can be lowered significantly in the presence of an external electric field, which can lead to incorrect conclusions about species concentration in the studied sample. This effect becomes more apparent with an increasing electric field, as a strong field lifts any adatoms away from the surface, weakening surface bonds. MD is used to study migration under high electric fields on short time scales (nanosecond timescale). Note that in order to simulate sufficiently fast atomic diffusion at low field, temperature high temperature is used. This temperature is comparable to peak temperature present in laser assisted field evaporation. An example is presented in figure 6, a 
single W atom was deposited "numerically" on the surface of a (011) atomic plane in a tip. The tip is then submitted simultaneously to high temperature and high voltage. This example is very similar to old experiments performed to study surface diffusion of ad-atoms in field ion microscopy, or more recent experiment to study tip temperature under laser illumination [26, 45]. It was experimentally observed that atoms tend to drift toward the edge of terraces when a sufficient field was present at the tip surface. It was interpreted as polarization effects in the gradient of the field on the extreme surface. MD simulations reproduce this behavior, showing that the surface electric field deforms the energy potential surface of the tip, diffusion being easier outward from the center of terraces. In addition, the surface electric field above the deposited atom tilts outward the center of the terrace when approaching the terrace border, giving rise to a driving force. Temperature in the model is artificially very high to reduce the computation time of such a diffusion. However, MD simulations can be used to calculate the value of the migration barriers under various field conditions, using e.g. the Nudged Elastic Band method, which can then be used as input for Kinetic Monte Carlo simulations. 


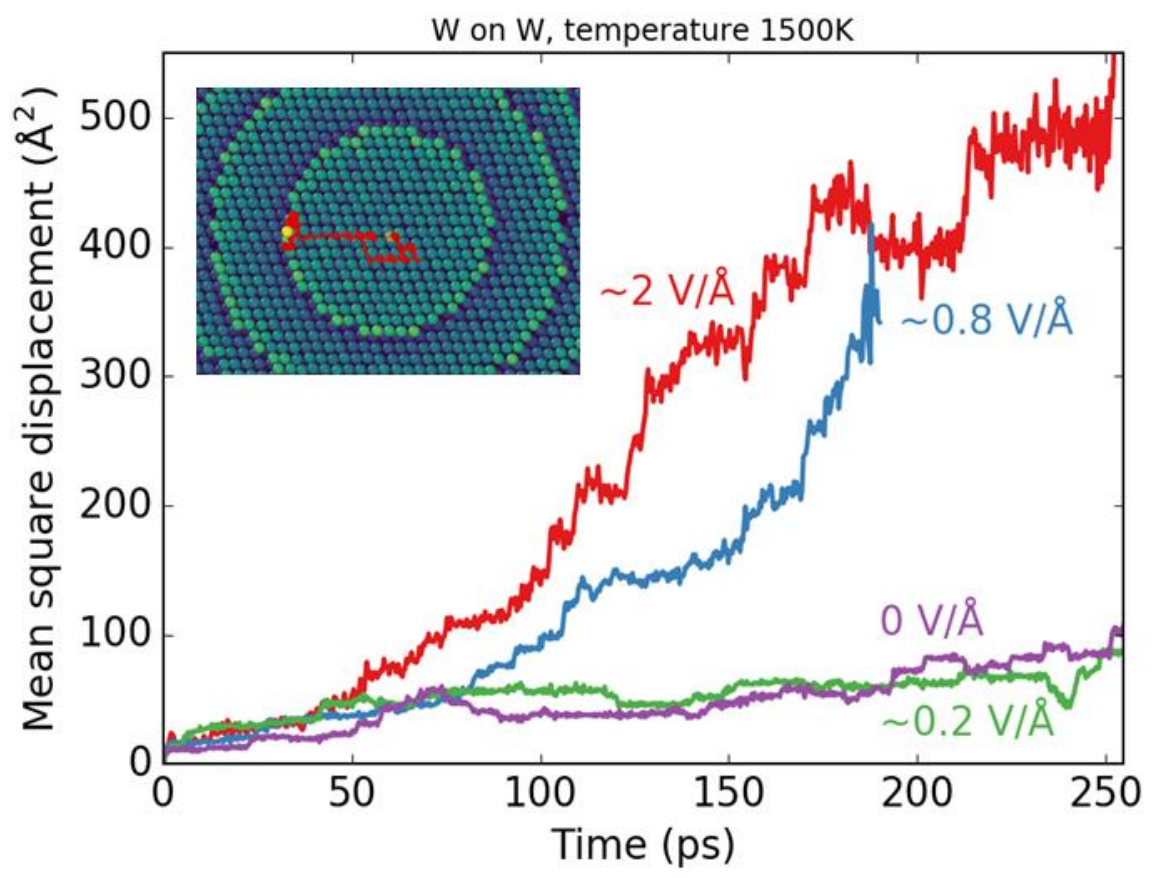

Figure 6: Average mean square displacement of a $W$ adatom on the apex of a $W$ sample exposed to high electric fields. It is seen that the mean square displacement increases faster at higher field values.

MD is also ideal for studying effects such as the "roll up" motion mentioned above. Using MD it is possible to examine the process at the atomic level, including variation on the process. For instance, it is seen that in some case, a protruding surface ad-atom near a ledge site, can sufficiently enhance the electric field, so that it triggers an atom to climb up from a lower ledge next to it. This indicates that the presence of mobile atoms on a surface can lead to a larger than expected decrease in the reliability of obtained atom probe data.

\section{Spatial degradation induced by molecular dissociation process}

When analysing metals, and especially pure metals, the measured spatial precision was found to be extremely good; the depth precision in the sub-Ångström range, and the lateral precision down to 0.2-0.3 $\mathrm{nm}$. The presence of species with different evaporation fields slightly perturb the positioning. Simulations shows that significant lateral and in-depth spread exist. For instance, simulating the ion trajectories of a 
disordered alloy $\mathrm{AB}$, evaporated assuming a moderate difference in evaporation field between $\mathrm{A}$ and $\mathrm{B}$ atoms $\left(\mathrm{E}_{\mathrm{B}}=1.2 \mathrm{E}_{\mathrm{A}}\right)$, the induced lateral spread was calculated to reach one interatomic spacing, in contrast with pure metals [46]. Addititionally, the sequence of evaporation in a random solid solution is not periodic. Layer after layer, the tip shape changes and does not remain self-similar, with each atom having a random local environment. Thus, the order of evaporation of atoms in equivalent crystallographic positions is not identical. This means that ion impacts of evaporated alloys will be subjected to a shift with respect to the ideal position observed in pure metals. Some additional effects are suspected to play a role in the evaporation of non-metallic specimens. For instance, in pure silicon, the depth precision of $1 \AA$ is close to that of metals, but it was noted that correlated evaporation of atoms and local rearrangement of the atomic planes could prevent the correct reconstruction of the diamond crystal structure [47]. As suggested by Kreuzer and co-authors, the crucial difference in field evaporation between metals and insulators is the basic fact that metals expel electrostatic fields and insulators allow them to penetrate [23, 48]. The tip's intense field shrinks the intrinsic band-gap of the insulator (the surface becomes semimetallic) and significantly reorganizes the structure of the atomic surface on the first atomic layers (the crystal structure will change much akin to melting so that site specificity could be lost). This local transformation disturbs the well-organized atomic structure of semiconductors and could give rise to additional deflections of ion trajectories. In oxides, the actual spatial precision is extremely perturbed. Thus, sharp features in reconstructed images appear blurred. For instance, in the analysis of $\mathrm{MgO}$ samples with embedded pure Au nanoparticles in APT, measured interfaces between $\mathrm{Au}$ and $\mathrm{MgO}$ matrix were found spread over several nanometres. However, in this system, abrupt interfaces were predicted theoretically and observed experimentally in electron microscopy and in field ion microscopy, [49,4]. This example clearly indicates the presence of issues in the analysis of oxides using APT.

One additional significant effect could be induced by the nature of the evaporation in these non-metallic samples. A significant contribution of the collected ions in the analysis of non-metals come from relatively 
simple molecules (more than $10 \%$ of the total number of events in oxides and nitrides). In addition, the emission of single atomic ions in experiments is often associated to correlated emission leading to multiple events, which can be observed in a correlated event map, where the mass-to-charge ratio of the second fragment is plotted versus the mass-to-charge ratio of the first fragment [50, 1-4]. In such a map, some specific features appear as correlation tracks, which have been explained as a result of the in-flight dissociation of a parent molecular ion into charged fragments $[1,51,52,53]$. Though the process is clearly identified, the origin of the in-flight dissociation of molecular dications has only been recently investigated from a theoretical point of view [20,21]. Charged molecules are found to be evaporated more easily in comparison to atomic ions in these materials. During the flight from the tip to the detector, ions experience a high electric field and charged molecules are not necessarily stable under this stress, and may fragment during flight (see figure 7). A theoretical analysis of the fragmentation of dicationic dimer for materials was performed for simple molecule often analyzed in atom probe experiments: $\left(\mathrm{ZnO}^{2+}, \mathrm{SiO}^{2+}\right.$, and also $\mathrm{C}_{2}{ }^{2+}$ ). The analysis is based on the simulation of the ion dynamics in the field of a paraboloid tip for a model where the internal energy of the molecule is deduced from accurate ab initio calculation of the electronic structure. 


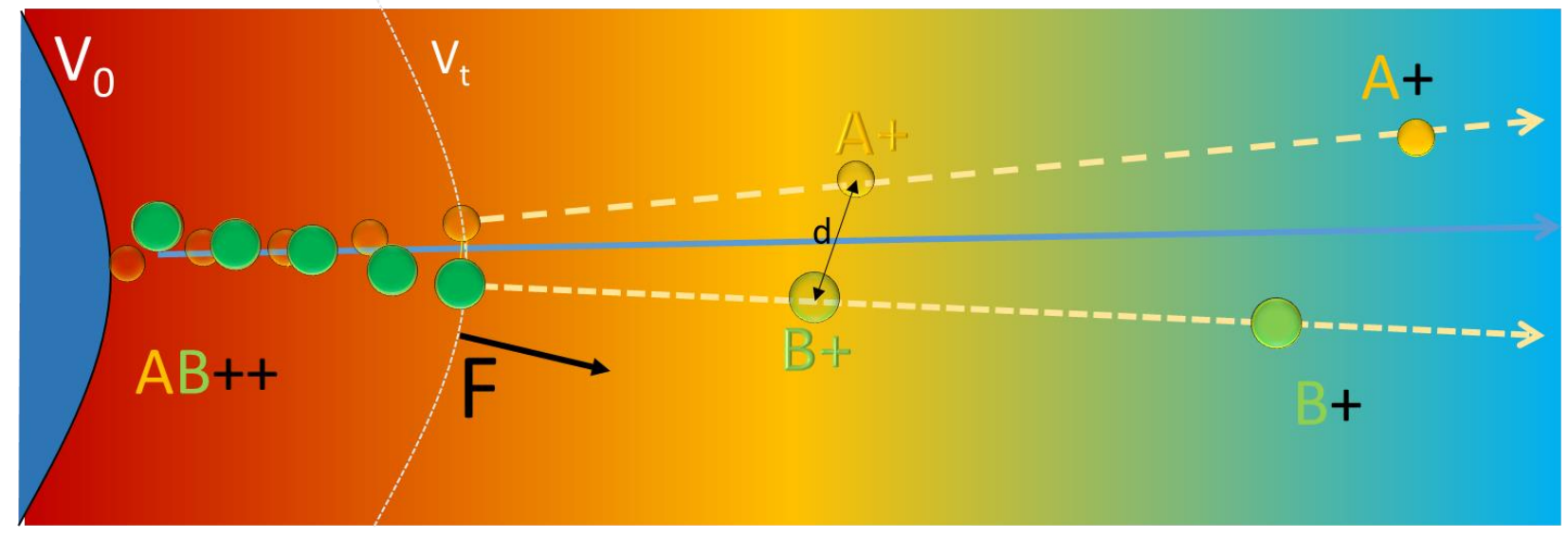

Figure 7: Schematic drawing of the dissociation of a single molecule AB in the space between the tip and the detector. Dissociation takes place after a lifetime $\tau$, the voltage, and the electric at this dissociation position being lower $\left(V_{t}\right)$ compared to the initial evaporated position.

We investigated the dynamics of dicationic metal-oxide molecules under large electric-field conditions, on the basis of ab initio calculations coupled to molecular dynamics. The electronic structure was obtained by means of a wave function approach for several ground and excited states of the molecular dications $[20,21]$. They were further parameterized with a convenient model Hamiltonian to obtain an efficient numerical model of the potential energy surfaces as a function of the dimer interatomic distance, the electric field magnitude and the orientation of the dimer with respect to the field. Applied to the case of $\mathrm{ZnO}^{2+}$ our simulation reveals the dissociation into three distinct exit channels (molecular $\mathrm{ZnO}^{2+}$, dissociation into $\mathrm{Zn}^{+} / \mathrm{O}^{+}$, dissociation into $\left.\mathrm{Zn}^{2+} / \mathrm{O}\right)$. The proportions of these channels depend critically on the field strength and on the initial molecular orientation with respect to the field. Under high electric field applied experimentally this dissociation takes place in the sub-picoseconds range, i.e. at the very early times of the dynamics.

Dissociation was found quite to be quite common for non-metal samples, for which the high electric field reduces the stability of dications in the ground state. For open-shell atoms and ions, the association of two 
fragments to form the dicationic dimer often leads to a copious set of potential energy curves (figure 8). For a quartz sample, generating $\mathrm{SiO}^{2+}$ dications, the above-described simulation shows that the lowest energy triplet states, including the ground states of the dication, are not stable under the experimental operating conditions used for quartz or amorphous sample analysis, with an electric field higher than 20 $\mathrm{V} / \mathrm{nm}$ (figure 8b). This instability is an intrinsic property of the $\mathrm{SiO}^{2+}$ dication, for which the energy barrier that prevents spontaneous dissociation disappears for an electric field of the order of $8 \mathrm{~V} / \mathrm{nm}$. However, we may note that excited states originating from the association of $\mathrm{Si}^{2+}\left({ }^{1} \mathrm{~S}\right)$ with $\mathrm{O}\left({ }^{1} \mathrm{D}\right)\left(1^{1} \Sigma\right.$ and $1^{1} \Pi$ in figure 8a) may survive to the fast field induced dissociation due to their deep potential wells located at short interatomic distances. The spontaneous dissociation of these singlet states in the $\mathrm{Si}^{+}\left({ }^{2} \mathrm{P}\right)+\mathrm{O}^{+}\left({ }^{4} \mathrm{~S}\right)$ asymptotic channel is prevented by a large energy barrier, and these states are stable under standard APT conditions. It was found that the spin-orbit coupling between electronic states of different spin symmetries is at the origin of the in-flight dissociation. We emphasize that this effect is only possible for excited states. For a given electronic state with a given spin symmetry, each bound ro-vibrational state contributes to the decay toward the ro-vibrational continuum of electronic states coupled by spin-orbit interaction to another spin symmetry. These kinds of couplings can be very efficient, leading to a broad range of lifetimes. In the case of $\mathrm{SiO}^{2+}$, the lifetime of such states obtained by means of first order perturbation theory was calculated in the range $10^{-11}-10^{-9} \mathrm{~s}$.

Such a lifetime is sufficient for the molecule to leave the high field region before splitting in two fragments. Each fragment thus shares a part of the kinetic energy acquired by the parent molecule during its acceleration in the tip potential before it breaks. This delayed dissociation process associated to excited states of molecules induces a direct correlation between the kinetic energies of each fragment as measured by the detection system (by time-of-flight mass spectrometry). This correlation, which can be highlighted using correlation maps of multiple hit events, is quite general and was observed for gallium nitride, 
aluminum nitride, silica, tungsten carbides, or different kind of oxides, for dimers as well as for larger clusters [50-53]. The fact that delayed dimer dissociation occurs at large distance from the tip where the electric field is significantly lower than at the tip surface led us to conclude that the observed lifetime is an intrinsic property of the emitted molecule, rather than a field induced effect. On the basis of the simulation, we can thus conjecture that non-dissociative excited electronic states are formed and then decay in a dissociative channel after some time. The origin of these excited states might be in the bond dissociation or in the laser pulse used to trigger evaporation.

(a)

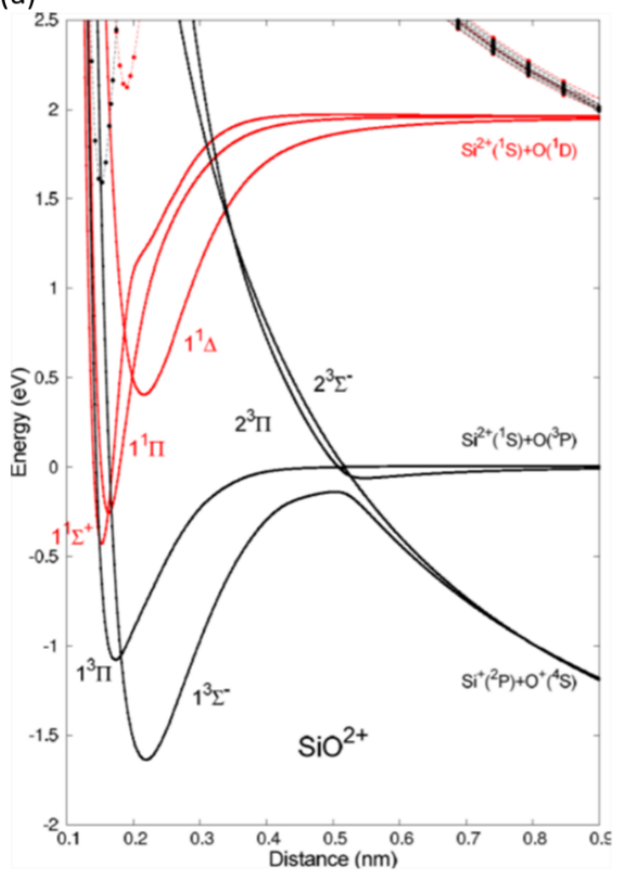

(b)

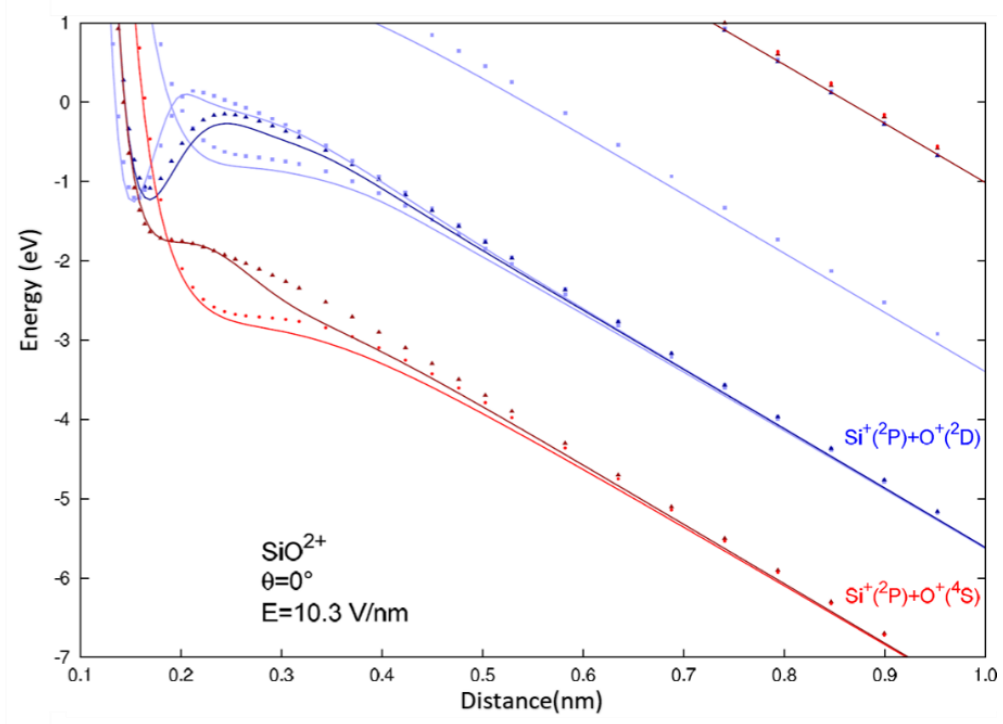

Figure 8: Lowest potential energy curves for $\mathrm{SiO}^{2+}$.(a) The red curves correspond to singlet states and the black curves correspond to triplet states for a molecule with no applied field (E=OV/nm). Higher energy states (dot lines in the figure) exist and are also computed but probability to have molecules excited to these levels is extremely small (b) Under high electric field $(E=10.3 \mathrm{~V} / \mathrm{nm}$, field in the direction of the axis of the molecule), ab-initio computation predicts field dissociation of lower energy states (red curves). Only existed states may survive to the quasi-instantaneous field dissociation (blue curves). 
Experimentally, dissociation is only evidenced by the correlation tracks in correlation histograms. We may note that the dissociations associated to the observed tracks are mainly produced by excited states of emitted molecules. Considering the low temperature of the sample surface, emission of ground state molecules is more probable, but in the case of $\mathrm{SiO}^{2+}$ these molecules are unstable in the high field region of the tip surface. Dissociation in this case is so fast that correlation tracks are not produced. In addition, this dissociation produces not only charged species but also neutral fragments emitted with low kinetic energy, most of them being lost in the detection process [20,52].

Dissociation has a clear effect on data quality for applications of atom probe tomography. Kinetic energy release of the order of a few $\mathrm{eV}$, induced by Coulomb repulsion between fragments adds a large uncertainty on the atoms final positions in the reconstruction. This was demonstrated experimentally in the dissociation of $(\mathrm{ZnO})_{2}{ }^{2+}[53]$. According to experimental data the distance between the impacts of dissociation products on the detector can be as large as $9 \mathrm{~mm}$, which, after three-dimensional reconstruction, corresponds to a distance of $6 \mathrm{~nm}$ on the tip surface whereas the atoms are known to originate from the same position. This dispersion is observed for fast or slow dissociation processes. A theoretical example is presented in figure 9. The spatial dispersion induced by field dissociation was simulated in the case of $\mathrm{SiO}^{2+}$, where the dynamics were simulated from the sample surface to a virtual detector located at $10 \mathrm{~cm}$. Molecules in different electronic states were placed at the surface of the sample, modelled in the paraboloidal approximation with a surface electric field of $\sim 11 \mathrm{~V} / \mathrm{nm}$ sufficient to easily dissociate ground state $\mathrm{SiO}^{2+}$. The dissociation plities were sampled from the computed spin-orbit coupling lifetimes for $4.10^{5}$ events and the corresponding trajectories of the fragment were determined up to the detector. Two dissociation cases and four different fragments are possible $\left(\mathrm{SiO}^{2+} \rightarrow \mathrm{Si}^{2+}+\mathrm{O} ; \mathrm{SiO}^{2+}\right.$ $\left.\rightarrow \mathrm{Si}^{+}+\mathrm{O}^{+}\right)$. The calculated distance between impacts, shown in figure 9 , accounts for several millimeters on the detector, (in good agreement with observed effects in some other oxides and carbides [52, 53,54 ]). 
The original positions of atoms are located at the barycenter of the detected pair. This distance is equivalent to a spatial precision on the tip of $1-2 \mathrm{~nm}$ for $\mathrm{Si}^{+} / \mathrm{O}^{+}$pairs and $1-5 \mathrm{~nm}$ for $\mathrm{Si}^{2+} / \mathrm{O}$ pairs. It is worth noting that dispersion is larger for $\mathrm{Si}^{2+} / \mathrm{O}$ dissociation, since conversely to $\mathrm{Si}^{2+}$, the $\mathrm{O}$ neutral fragment is not accelerated by the remaining electric field in vacuum that amplifies the distance between impacts on the detector.

The measured amount of molecules decreases with increasing applied field when analyzing semiconductors, or non-metallic materials in APT. The probability to emit single ions in high fields might be higher, but we must keep in mind that molecules are rapidly broken under the presence of the high electric field. Since most of the molecules produced by field evaporation are probably broken during the flight, the lateral precision will be degraded to several nanometers when analyzing oxides or nitrides. Degradation will be amplified with fast dissociation. It is also worth noting that due to the intrinsic detector efficiency ( $37 \%$ to $80 \%$ ), most of the dissociated ion pairs are collected as single hits. In addition, fast dissociations produce many neutral oxygens from $\mathrm{SiO}^{2+}$ and $\mathrm{ZnO}^{2+}$ and, therefore, the global detection efficiency is reduced. It also biases the measurement of composition in APT, since these neutral species are at best incorrectly positioned in the mass spectra, if not completely undetected. 

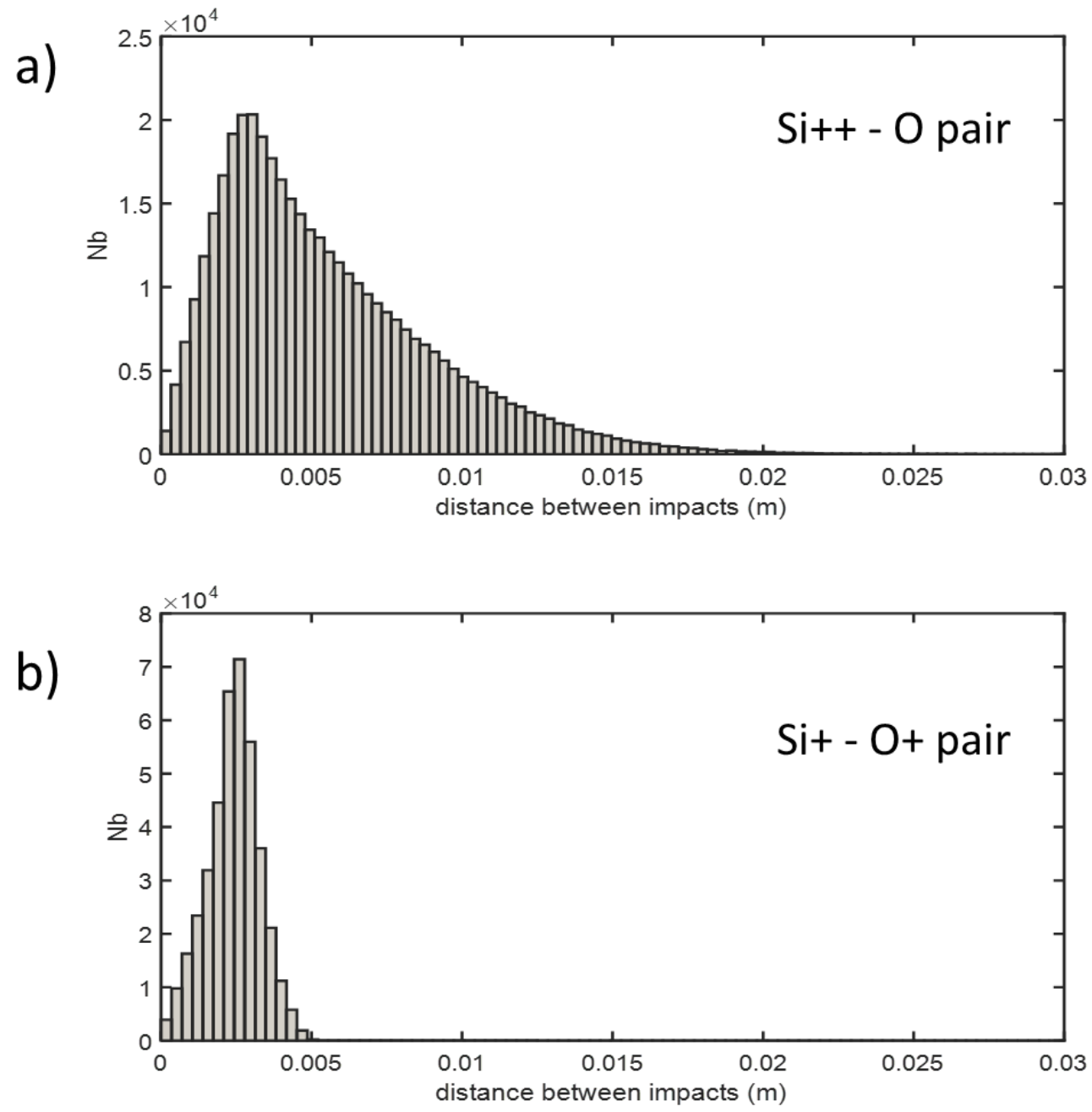

Figure 9: Calculated distance between impacts for fragments spatial dispersion for fragment of dissociation in the reaction $\mathrm{SiO}^{2+\rightarrow} \mathrm{Si}^{+}+\mathrm{O}(\mathrm{a})$ and $\left.\mathrm{SiO}^{2+\rightarrow} \mathrm{Si}^{+}+\mathrm{O}^{+}(b)\right)$. Histograms were calculated through molecular dynamic simulation of the dissociation of $4.10^{5}$ events from the tip to the detector for an electric field magnitude of $11 \mathrm{~V} / \mathrm{nm}$ applied to the sample..

\section{Conclusion and outlook}

Atom Probe Tomography is a must-have in the panel of instruments for materials analysis. The inherent performance of this three-dimensional nano-characterization tool in terms of spatial positioning of local features (segregation, clusters, local composition fluctuations, etc..) excites the interest of any 
beginner in APT. In this paper, it is detailed how the spatial precision, one of the key-qualities of the technique, could be strongly degraded by different physical mechanisms. The atomic scale precision, associated with the analysis of pure metals, tends to be lost when non-optimal analyzing conditions are used. It is particularly true when materials with non-metallic bond are probed using APT. The precision is degraded by complex physical processes acting on the analyzed sample surface. The in-depth evaluation of the ultimate performances that can be achieved in these cases requires a deep knowledge of the physical principles in action in the instrument. Recent modeling approaches were developed with the aim to give interpretation to the joint effect of the electric field, the temperature, and the physical properties of the object of interest. The ideal view of the picking process induced by field evaporation is to be considered with care to evaluate any subtle movement of atoms. Semi-classical approaches that have modelled field evaporation by KMC models, or MD models assuming simple inter-atomic potentials give a good interpretation of observed spatial dispersions. However, in oxides or nitrides, these models are clearly insufficient since local bonds between metallic and non-metallic elements are extremely sensitive to the presence of the external field. We detailed a simple example including how field evaporated molecules can dissociate, generating a significant blurring effect in the projected image. This article was focused on the spatial precision. It may be noted that the same models can be used to understand artefacts in composition measurements, or image reconstruction accuracy. We also think that improving simulation models will in the future be a necessary step to better control the analysis of a material. The main limitation today is the cumbersome computation time required by these approaches, but progresses will necessarily be achieved in the future. 


\section{ACKNOWLEDGEMENTS}

FV acknowledges the financial support of the EMC3 Labex, AQURATE project and DYNAMITE project, and the support from CAMECA, RINGAPT2 project. FD and SP acknowledge the funding by Academy of Finland (Grant No. 269696).

\section{Data availability}

The raw/processed data required to reproduce these findings cannot be shared at this time due to technical or time limitations. However, the data that support the findings of this paper are available from the corresponding author on reasonable request. 


\section{$\underline{\text { References : }}$}

1 B. Gault, M.P. Moody, J.M. Cairney, S.P. Ringer, Atom Probe Microscopy, Springer New York, New York, NY, 2012.

2 D. Larson, T. Prosa, T. Kelly, Local Electrode Atom Probe Tomography - A user's guide, Springer New York, 2013.

$3 \quad$ M.K. Miller, R.G. Forbes, Atom-Probe Tomography, Springer US, Boston, MA, 2014.

4 W. Lefebvre-Ulrikson, F. Vurpillot, X. Sauvage, Atom Probe Tomography, From theory to practice, Academic Press, London, UK, 2016

$5 \quad$ S .T. Loi, B. Gault, S.P. Ringer, D.J. Larson, B.P. Geiser, Ultramicroscopy. ; 132:107-13. doi:0.1016/j.ultramic.2012.12.012. (2013)

6 Oberdorfer, C.; Eich, S.M.; Schmitz, G., Ultramicroscopy Volume: 128 Pages: 55-67(2013), doi: 10.1016/j.ultramic.2016.03.008

7 N Rolland, F Vurpillot, S Duguay, D Blavette, Microscopy and Microanalysis 21 (06), 16491656 (2015), DOI: $10.1017 /$ S1431927615015184

8 F. Vurpillot and C. Oberdorfer, Ultramicroscopy 159, 202-216, (2015) DOI:

10.1016/j.ultramic.2014.12.013

9 M. Gruber, F. Vurpillot, A. Bostel, B. Deconihout, Surface Science, Volume 605, Issues 23-24, Pages 2025-2031, (2011), DOI: 10.1016/j.susc.2011.07.022

10 D. Haley, M.P. Moody, G. D. W. Smith, D. ,Level Set Methods for Modelling Field Evaporation in Atom Probe, MICROSCOPY AND MICROANALYSIS Volume: 19 Issue: 6 Pages: 1709-1717, (2013), DOI : $10.1017 /$ S1431927613013299

11 O. Dimond, Modelling the Reconstruction of 3D Atom Probe Data. University of Oxford, (1999).

12 Niewieczerzal, D.; Oleksy, C.; and Szczepkowicz, S. Multi-scale simulations of field ion microscopy images - Image compression; Ultramicroscopy 112; pp. 1-9 (2012), DOI: 10.1016/j.ultramic.2011.10.007

13 Oberdorfer, Christian; Schmitz, Guido, On the Field Evaporation Behavior of Dielectric Materials in Three-Dimensional Atom Probe: A Numeric Simulation, MICROSCOPY AND MICROANALYSIS Volume: 17 Issue: 1 Pages: 15-25 (2011), DOI: 10.1017/S1431927610093888 
14 Geiser, BP ; Larson, DJ; Gerstl, SSA ; Reinhard, D ; Kelly, TF ; Prosa, TJ ; Olson, JD,A System for Simulation of Tip Evolution Under Field Evaporation; MICROSCOPY AND

MICROANALYSIS; Volume: 15, Pages: 302-303, Supplement: 2, (2009), DOI:

\section{$10.1017 / \mathrm{S} 1431927609098298$}

15 Marquis, EA, Geiser, BP ; Prosa, TJ; Larson, DJ, Evolution of tip shape during field evaporation of complex multilayer structures, JOURNAL OF MICROSCOPY Volume: 241 Issue: 3, Pages: 225-233 (2011), DOI: 10.1111/j.1365-2818.2010.03421.x

16 Vurpillot, F; Bostel, A; Blavette, D, Trajectory overlaps and local magnification in threedimensional atom probe, APPLIED PHYSICS LETTERS Volume: 76 Issue: 21 Pages: 3127-3129, (2000), DOI: 10.1063/1.126545

17 Atomistic Simulations of Surface Effects Under High Electric Fields, S Parviainen, M Dagan, S Katnagallu, B Gault, M Moody, F Vurpillot, Microscopy and Microanalysis 23 (S1), 644-645 (2016);

DOI: $\quad$ 10.1017/S1431927617003889

18 Djurabekova F1, Parviainen S, Pohjonen A, Nordlund K.,Phys Rev E Stat Nonlin Soft Matter Phys.;83(2 Pt 2):026704. Epub 2011 Feb 14., Atomistic modeling of metal surfaces under electric fields: direct coupling of electric fields to a molecular dynamics algorithm. (2011); DOI:

10.1103/PhysRevE.83.026704

19 Parviainen, S., et al. Atomistic simulations of field assisted evaporation in atom probe tomography Journal of Physics D: Applied Physics 49.4: 045302. (2015), DOI: doi.org/10.1088/0022$3727 / 49 / 4 / 045302$

20 D. Zanuttini, I. Blum, L. Rigutti, F. Vurpillot, J. Douady, E. Jacquet, P.-M. Anglade, and B. Gervais, Electronic structure and stability of the $\mathrm{SiO} 2+$ dications produced in tomographic atom probe experiments, The Journal of Chemical Physics 147, 164301 (2017); https://doi.org/10.1063/1.5001113 21 David Zanuttini, Ivan Blum, Lorenzo Rigutti, François Vurpillot, Julie Douady, Emmanuelle Jacquet, Pierre-Matthieu Anglade, and Benoit Gervais, Simulation of field-induced molecular dissociation in atom-probe tomography: Identification of a neutral emission channel, Phys. Rev. A 95, 061401(R) , (2017), DOI: 10.1103/PhysRevA.95.061401

22 F Vurpillot, B Gault, BP Geiser, DJ Larson, Reconstructing atom probe data: A review, Ultramicroscopy 132, 19-30 (2012); DOI: 10.1016/j.ultramic.2013.03.010

23 M. Karahka, H.J. Kreuzer, Ultramicroscopy,Volume 132, September 2013, Pages 54-59 ; DOI : 10.1016/j.ultramic.2012.10.007 
24 Chen Y.C. and Seidman D.N., Surface Science, 27, 231, (1972), DOI: 10.1016/00396028(71)90031-8

25 F Vurpillot, G Da Costa, A Menand, D Blavette, Structural analyses in three- dimensional atom probe: a Fourier transform approach, Journal of Microscopy 203 (3), 295-302 (2001); DOI: 10.1046/j.1365-2818.2001.00923.x

26 T.T. Tsong, Atom Probe Field Ion Microscopy, Cambridge University Press, NY, USA, (1990)

27 B. Gault, M. Müller, A. La Fontaine, M. P. Moody, A. Shariq, A. Cerezo, S. P. Ringer, and G. D. W. Smith, Journal of Applied Physics 108, 044904 (2010); https://doi.org/10.1063/1.3462399 28 M. Wada, On The Thermally Activated Field Evaporation Of Surface Atoms, JOURNAL DE PHYSIQUE,Colloque C9, supplément au n012, Tome 45, pages C9-89 (1984); DOI:

10.1051/jphyscol:1984916>

29 A. R. Waugh, E. D. Boyes, and M. J. Southon, Surf. Sci. 61, 109 (1976)

30 Sanchez C.G., Lozovoi A.Y., Alavi A., Field-evaporation from first-principles, Molecular Physics, 102, No. 9-10, pp. 1045-1055 (2004)

31 B. Gault, F. Danoix, K. Hoummada, D. Mangelinck, H. Leitner, Impact of directional walk on atom probe microanalysis, Ultramicroscopy, Volume 113, February 2012, Pages 182-191

32 W. Lefebvre, A. Loiseau, A. Menand, Ultramicroscopy, Volume 92, Issue 2, July 2002, Pages 77-87 (2002) ; DOI : 10.1016/S0304-3991(02)00070-0

33 S Parviainen, F Djurabekova, S P Fitzgerald, A Ruzibaev and K Nordlund, Atomistic simulations of field assisted evaporation in atom probe tomography, Journal of Physics D: Applied Physics, Volume 49, Number 4, (2015) DOI: 10.1088/0022-3727/49/4/045302

34 EA Marquis, F Vurpillot, Chromatic aberrations in the field evaporation behavior of small precipitates

Microscopy and microanalysis 14 (6), 561-570, (2002); DOI /10.1017/S1431927608080793

35 F Vurpillot, A Bostel, E Cadel, D Blavette, The spatial resolution of 3D atom probe in the investigation of single-phase materials, Ultramicroscopy 84 (3-4), 213-224 (2002); DOI: 10.1016/S0304-3991(00)00035-8

36 Joaquín Peralta, Scott R. Broderick, Krishna Rajan,Mapping energetics of atom probe evaporation events through first principles calculations, Ultramicroscopy, Volume 132, Pages 143-151 (2013); DOI: 10.1016/j.ultramic.2013.02.007 
37 T. Ono, T. Sasaki, J. Otsuka, K. Hirose, First-principles study on field evaporation of surface atoms from W(0 11 1) and Mo(0 1 1) surfaces, Surface Science, Volume 577, Issue 1, Pages 42-46, (2005); DOI: 10.1016/j.susc.2004.12.024

38 L.C. Wang, H.J. Kreuzer, Kinetic theory of field evaporation of metals, Surface Science, Volume 237, Issues 1-3, Pages 337-346 (1990); DOI: 10.1016/0039-6028(90)90546-K

39 Edward R. McMullen and John P. Perdew, Theory of field evaporation of the surface layer in jellium and other metals, Physical Review B VOLUME 36, NUMBER 5, (1987); DOI:

10.1103/PhysRevB.36.2598

40 Müller, Erwin W. "Field desorption." Physical Review 102.3: 618, (1956); DOI:

10.1103/PhysRev.102.618

41 M. Veske, A. Kyritsakis, K. Eimre, V. Zadin, A. Aabloo and F. Djurabekova; Dynamic coupling of a finite element solver to large-scale atomistic simulations; submitted for publication to Journal of Computational Physics; https://arxiv.org/abs/1706.09661]

42 A. Kyritsakis, M. Veske, K. Eimre, V. Zadin, F. Djurabekova; Thermal runaway and evaporation of metal nano-tips during intense electron emission; submitted for publication to Journal of Physics D: Applied Physics; https://arxiv.org/abs/1710.00050

43 Pohjonen, A. S., et al. "Dislocation nucleation on a near surface void leading to surface protrusion growth under an external electric field." Journal of Applied Physics 114.3 (2013): 033519. 44 M. Dagan, B. Gault, G.D.W. Smith, P.A.J. Bagot, and M.P. Moody, autommated Atom-byAtom Three-Dimensional (3D) Reconstruction of Field Ion Microscopy data, Microscopy And Microanalysis, Volume 23, 2, pp.255-268 (2017); https:Ildoi.oreg/10.1017/S1431927617000277

45 F Vurpillot, J Houard, A Vella, B Deconihout, Thermal response of a field emitter subjected to ultra-fast laser illumination, Journal of Physics D: Applied Physics 42 (12), 125502 (2009); DOI: $10.1088 / 0022-3727 / 42 / 12 / 125502$

46 F Vurpillot, A Bostel, E Cadel, D Blavette, The spatial resolution of 3D atom probe in the investigation of single-phase materials, Ultramicroscopy 84 (3), 213-224 (2000);

DOI: $10.1016 / \mathrm{S} 0304-3991(00) 00035-8$

47 E Cadel, F Vurpillot, R Lardé, S Duguay, B Deconihout, Depth resolution function of the laser assisted tomographic atom probe in the investigation of semiconductors, Journal of Applied Physics 106 (4), 044908 (2009); DOI : 10.1063/1.3186617 
48 EP Silaeva, L Arnoldi, ML Karahka, B Deconihout, A Menand, HJ Kreuzer, A. Menand, Do dielectric nanostructures turn metallic in high-electric dc fields? Nano letters 14 (11), 6066-6072 (2014); DOI: $10.1021 / \mathrm{nl} 502715 \mathrm{~s}$

49 A Devaraj, R Colby, F Vurpillot, S Thevuthasan, The journal of physical chemistry letters 5 (8), 1361-367 (2014); DOI: 10.1021/jz500259c

50 D.W. Saxey, Correlated ion analysis and the interpretation of atom probe mass spectra, Ultramicrosc. 111, 473-479, (2011).; DOI: 10.1016/j.ultramic.2010.11.021

51 F. Aquilante, L. De Vico, N. Ferre, G. Ghigo, P.-A. Malmqvist, P. Neogrady, T. B. Pedersen, M.

Pitonak, M. Reiher, B. O. Roos, L. Serrano-Andr'es, M. Urban, V. Veryazov, and R. Lindh, J. Comput. Chem. 31, 224 (2010); DOI : 10.1002/jcc.21318.

52 B Gault, DW Saxey, MW Ashton, SB Sinnott, AN Chiaramonti, MP Moody, Behavior of molecules and molecular ions near a field emitter, New Journal of Physics 18 (3), 033031 (2016); DOI:

10.1088/1367-2630/18/3/033031/meta

53 I Blum, L Rigutti, F Vurpillot, A Vella, A Gaillard, B Deconihout, Dissociation dynamics of molecular ions in high DC electric field, The Journal of Physical Chemistry A 120 (20), 3654-3662 (2016); DOI: 10.1021/acs.jpca.6b01791

54 Zirong Peng, Francois Vurpillot, Pyuck-Pa Choi, Yujiao Li, Dierk Raabe, On the detection of multiple events in atom probe tomography, Baptiste Gault, Ultramicroscopy, Volume 189, June 2018, Pages 54-60 (2018) ; https://doi.org/10.1016/j.ultramic.2018.03.018 